\title{
Stress, Pressure and Fatigue on Aircraft Maintenance Personal
}

\author{
Luis F. F. M. Santos ${ }^{1,2}$, R. Melicio ${ }^{2,3,4}$
}

\begin{abstract}
This paper focuses on an analysis of the stress, the pressure and the fatigue as part of the Dirty Dozen and Human Factors procedures. An online international survey has been carried out to ascertain the professional levels of the fatigue, the stress and their pressure exposure. This work is a contribution to the aeronautical safety in order to alert authorities about the stress, the pressure and the fatigue that aircraft maintenance personnel suffers. Safety is the main driver in aviation related professions. Maintenance related personnel is constantly subjected to several external circumstances that might originate errors in the performance or evaluation in maintenance related tasks. Authorities have clearly regulated flight crew and air traffic controllers working and resting periods, but maintenance personnel regulations do not reflect the same procedures. The Aeronautical industry 4.0 with the upcoming digital transformation will increase the safety margin and it will reduce the aircraft maintenance ground time. Copyright (C) 2019 Praise Worthy Prize S.r.l. - All rights reserved.
\end{abstract}

Keywords: Maintenance, Human Factors, Stress, Fatigue, Pressure, Aviation, Annex 19, Dirty Dozen, Part-145, Part-M, Safety

\section{Nomenclature}

MOM

EASA

ICAO

FAA

LAM

AOG

SMS

FRMS

\author{
Maintenance Organization Manual \\ European Aviation Safety Agency \\ International Civil Aviation Organization \\ Federal Aviation Administration \\ Linhas Aéreas de Moçambique \\ Aircraft on Ground \\ Safety Management System \\ Fatigue Risk Management System
}

\section{Introduction}

Air transport is considered to be the safest method of transport [1], [2], due to the close interaction between aeronautical authorities, aviation industry (aircrafts), operators and aircraft maintenance industry [3]. As aircrafts become more reliable, humans have played a progressively more important causal role in aviation accidents [4]. Hence, aviation safety is the major concern and it is one of the principal drive factors of the civil aviation industry and regulamentary purposes. Since the beginning of air transport, the need of rules and regulations have driven the aviation industry towards safer, and more reliable technologies and procedures.

The aircraft maintenance is a complex system, that requires sustained coordination, communication and cooperation between different work groups and teams including the aircraft maintenance and the airline, in order to ensure safety and efficient operations (minimizing error) [5]. Aircraft maintenance is highly regulated, safety critical, complex and competitive. There is the need to develop innovative solutions to address process efficiency without compromising safety and quality [5], particularly with regard to the work hours of maintenance personnel. It is not proper to talk about safety if the the Flight Crew rests for 12 hours while the maintenance team was works 16 hours or more. In [6] the aviation safety depends on minimizing error in all the facets of the system. While the role of flight deck human error has always received much emphasis, recently, more attention has been directed towards reducing human error in maintenance and inspection. Aviation maintenance and inspection tasks are part of a complex organization, where individuals perform different tasks in an environment with time pressures, sparse feedback, and sometimes difficult ambient conditions. These situational characteristics, in combination with generic human erring tendencies, result in different forms of error. In [7] a study on the mental workload in pilots is conducted. It shows a method to evaluate the mental distress and its affect on the eye pupils. This study has brought new insights to the pilot's performance during high mental workload. In [5] authors have also confirmed that aircraft maintenance is a highly regulated, safety critical, complex industry currently facing unprecedented challenges. Pressure is on aircraft manufacturers, from their customers, to design aircraft with pushed out maintenance schedules. The first key factors to improve flight safety has been introduced by ICAO, and it leads the industry to the development of methods to eliminate the accidents/incident common causes, such as the introduction of better technology, aircraft redundancies and systems compartmentalization and segregation. With these developments, together with organizational and human factors, the aviation industry has performed an 
increase of safety until it has become as it is known today: the safest way to travel. This improvement, after the technological ones, has been achieved mainly due to very tight regulations that have introduced new procedures, limitations and standard processes. The second key factor has been the introduction of regulations to eliminate the random and multiple causes of the safety issues. Here, the aviation industry still has a long path to go, being 2017 the safest year ever for aviation, might be a good indicator that the industry is on the right track, but it still has to face several improvements and challenges to reach the mark of zeros accidents and zero incidents. The fatal airline accidents between 1946-2017 [8] are shown in Fig. 1.

Fig. 1 presents the downtrend of the number of fatal airliner accidents; these numbers encourage all the aviation professionals and they provide good indicators about the application status of all the regulations nevertheless the industry must adopt the strategy that "one accident is already too much". This paper is a contribution on the analysis of the stress, the pressure and the fatigue on aircraft maintenance personnel as part of the Dirty Dozen and Human Factors procedures. An online international survey has been carried out to ascertain the professional levels of the fatigue and the stress and their pressure exposure. The paper is structured as follows. Section II presents the current regulamentary system. Section III presents the need for regulations. Section IV presents the international survey. Section V presents the hazard procedures. Section VI presents the diagnostics and recommendations. Finally, Section VII outlines the conclusion.

\section{Current Regulamentary System}

All over the several industry divisions, such as but not limited to:

- Design;

- Manufacturing;

- Maintenance Management;

- Maintenance Technicians Licensing;

- Maintenance Personal Training;

- Maintenance Shops;
- Air Traffic Control;

- Air Traffic Control Licensing;

- Aircrew Training and Licensing;

- Air Operations;

- Aerodromes;

- Rules of the Air;

have suffered heavy regulations which have led to both the standardization and to an increase of safety. One of the major safety increment drive factors has been the introduction of Human Factors considerations [9].

Human Factors are taken very seriously by EASA; in this European organization there is dedicated personnel that studies human behavior under several conditions. Several studies have been made, and an entire new field of study dedicated only to human factors exists. In fact, these studies are so important that FAA and ICAO also have dedicated teams on these analyses [9], [10]. ICAO has issued Annex 19, Safety Management System, which has led to the worldwide authorities issuing its own regulations. Annex 19 is divided into two major phases [9]:

phase 1:

- Technical: Better Technology.

- Human: Human Factors Management; Crew Resources Management; Fatigue Risk Management.

- Organizational: Quality Assurance; Quality Management System; Threat and Error Management. phase 2:

- Safety Management System.

- State Safety Program. The ICAO Annex 19 Roadmap [9] is shown in Fig. 2. Positioning on the European regulations, and on Fatigue Risk Management, EASA has created for Flight Crew under Part-ORO.FTL.210 the maximum duty limitations [9]:

Duty Periods:

- 190 Duty hours in any 28 consecutive days;

- 110 Duty hours in any 14 consecutive days;

- 60 Duty hours on 7 consecutive days.

Flight Time:

- 100 Flight hours in any 28 consecutive days;

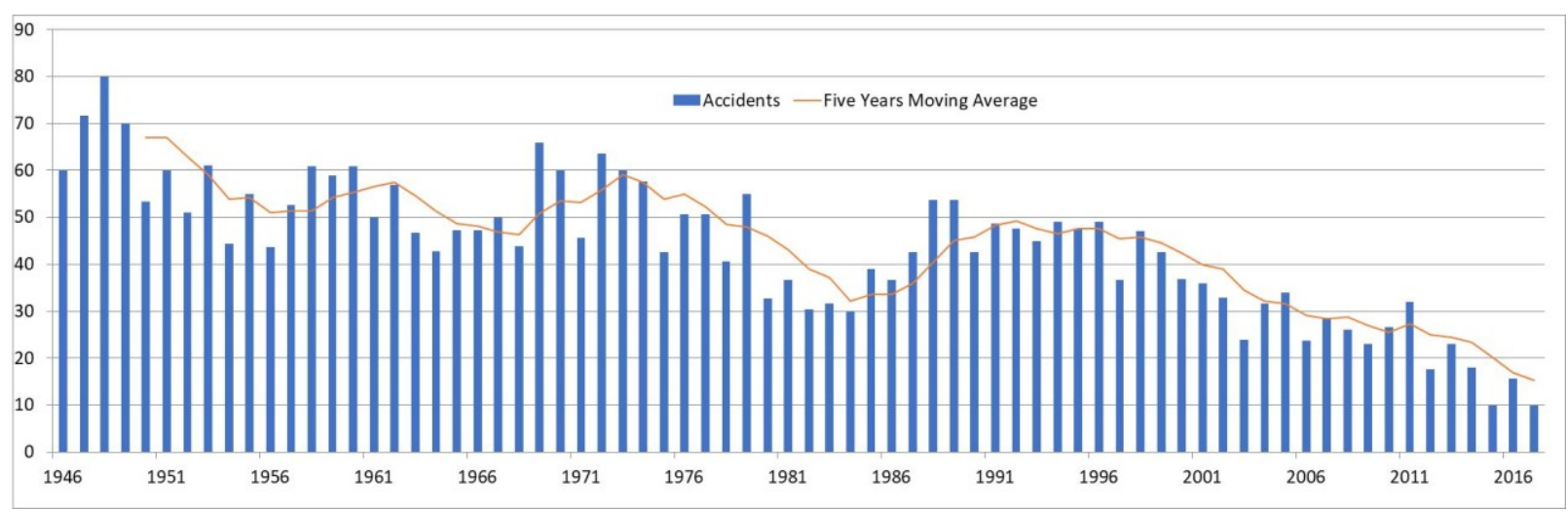

Fig. 1. Fatal airline accident 1946-2017 
- 900 Flight hours in any calendar year;

- 1000 Flight hours in any 12 consecutive months.

Also, on Part-ORO.FTL.205, [11] created the maximum daily flight duty period of $13 \mathrm{~h}$, followed by a minimum rest period of $12 \mathrm{~h}$ under Part-ORO.FTL.235 [11].

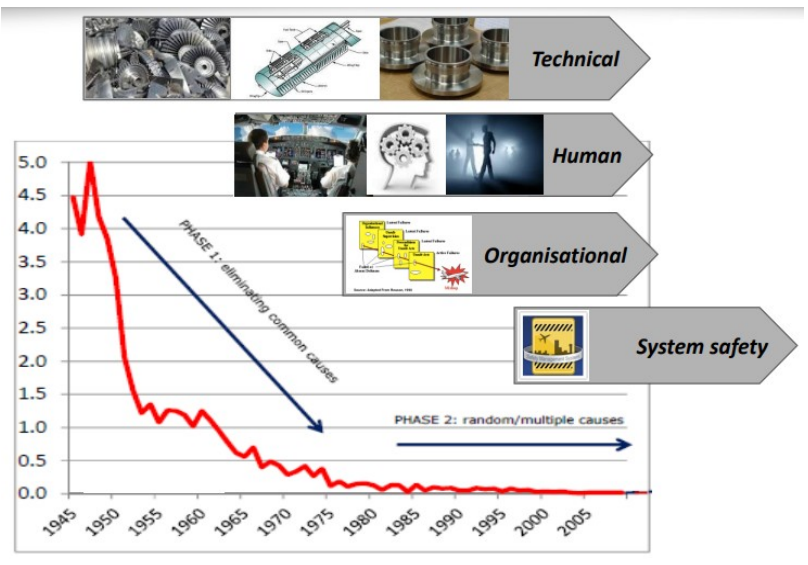

Fig. 2. ICAO Annex 19 Roadmap [9]

Also Air Traffic Control personnel has its working time regulated:

- 2 consecutive hours on tower duty;

- 1.5 consecutive hours on airport flight approach duty;

- One 8 hours shift;

- 2 consecutive rest days after three consecutive 8 hours shifts.

It can be verified that EASA has clearly regulated both aircrew and air traffic control personnel, nevertheless, the maintenance personnel, both EASA Part-M and Part-145 does not refer any workload limit for these aircraft maintenance professionals [12],[13]. The only mention to Human Factors is made on Part-145 145.A.47 that states that the maintenance organization must have into account the limitations of human performance. As it can be verified, no additional work limit and time limitations are given, referring only to the maintenance shops MOM [12].

\section{The Need for Regulations}

There are clearly some key indicators which demand regulations on maintenance personnel. As it can be seen forward on Chapter IV, there are some indicators that regulators must pay special attention to. On November 29 th of 2013, the pilot from LAM committed suicide with everyone onboard. On March 24th of 2015, the first officer from Germanwings also took the same action as the LAM pilot [14]. Unfortunately, there are many more cases; there is an urgent need to stop possible future similar cases. Passing through the accident root causes, there is the need to identify on early stage the psychological symptoms that might lead to the prevention and the mitigation of similar situations. These examples are taken from the obvious, because they were intentional and they are the tip of the iceberg. The remaining aviation areas, focusing on maintenance, are subjected to an enormous pressure, stress and fatigue.

The market demands for professionals that are available $24 \mathrm{~h} / 7$ days and professionals that dedicate an important part of their daily life to the industry. It Is common that maintenance managers demand a significant percentage of overtime hours to keep the operations and scheduled lead times, by doing this also cut personnel expenses by reducing the necessary personnel to keep their operations running. In a study made by Boeing, pointed aircraft maintenance is the root cause of $15 \%$ of commercial accidents happened between 1982 and 1991 [15]. Later on, back on 1994 Dupont, G realized that quite a significant share of the maintenance related accidents has been routed to only 12 main causes, they are called the "Dirty Dozen" [16]. Aircraft maintenance personnel today still learns these Dirty Dozen, which are part of the recurrent mandatory training under the Human Factors course. Stress, pressure and fatigue are among this twelve of the most common human error preconditions.

Now, 20 years have passed, and still the regulations don't reflect clear measures to mitigate this twelve dirty practices and behaviors. The stress, the pressure and the fatigue have several sources; some of them can be selfinduced, other ones come from company conditions and market pressure.

The stress, the pressure and the fatigue can be divided into 2 families and 4 groups [17]. The workplace and personal factors contributing to employee fatigue [10],[18], as shown in Fig. 3 are:

- Work factors: Workload breaks; Type of work; Work duration; Regulations; Team climate; Staffing.

- Personal Factors: Sleep quality; Circadian rhythms; Sleep duration; Socio-economic factors; Commuting; Family life; Social life.

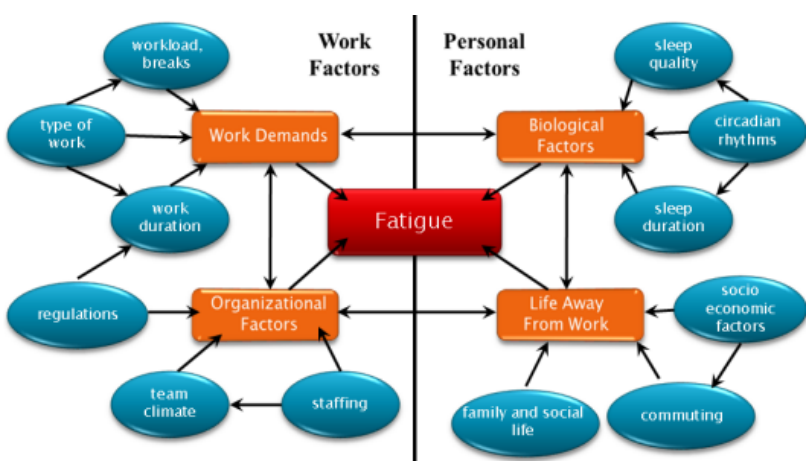

Fig. 3. Workplace and personal factors contributing to employee fatigue [10]

There are also hidden factors which are quite common to be referred in close statistics but they are not admitted by personal interviews [19]:

- Work demands;

- Hierarchy pressure;

- Time pressure and economic pressure. 
Including human factors training in the aerospace industry it is already demanded by some regulators; nevertheless, industry must adopt more proactive measures to improve the human factors disciplines [20]:

- Industrial Engineering;

- Clinical Psychology;

- Experimental Psychology;

- Educational Psychology;

- Organizational Psychology;

- Medical Sciences;

- Computer Sciences;

- Cognitive Sciences;

- Safety Engineering;

- Anthropometric Sciences.

Through the evolution of aircraft technology and reliability, the technical causes for the aircraft incident have decreased to unprecedent levels; however, human causes have taken the main cause of accidents which nowadays is near $80 \%$ of the total accident root causes [8] is shown in Fig. 4.

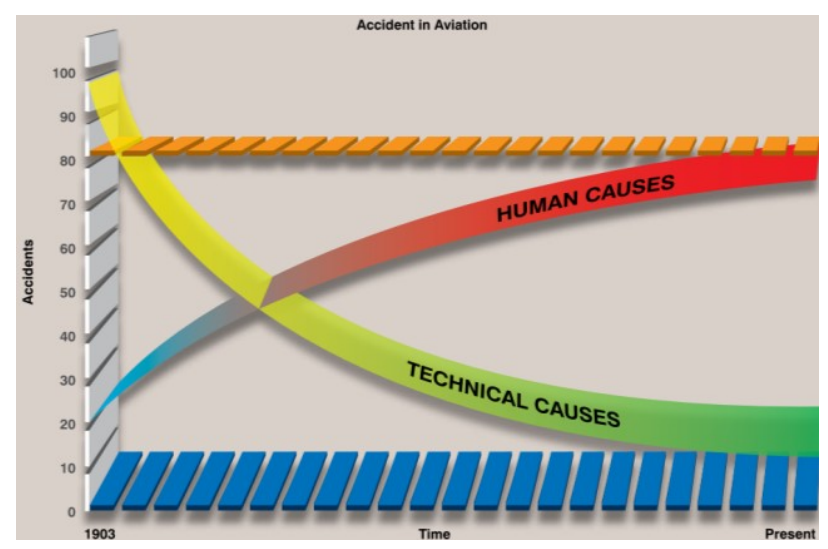

Fig. 4. Evolution of root causes in aviation accidents [6]

In aviation, the workload is known to be quite demanding, specially the Line and Heavy maintenance, which the related personnel is subjected to an enormous quantity of requests and tasks at the same time; this factor, if not handled with care, might lead to loss of information, or more seriously, missing maintenance tasks [19].

Hierarchy pressure to perform some type of assessment instead of other, or also to extend the working schedule is also pointed by the statistics to be another cause of Stress/Fatigue.

The time pressure is the amount of time to perform an excessive number of tasks; this might also lead to errors [19].

Economic pressure is when economic factors are put in front of safety factors; also, this is admitted on surveys but not in personal interviews [19].

In [21] authors affirm that unintentional human errors in aircraft maintenance, occurs all the time.

According to the definition, human factor is an unintentional error in the work which results in immediate damage of the system or it may be a hidden error which represents a potential danger for the technical airworthiness of the aircraft.

As shown in Fig. 5, there will be a trend of increase in the human error if no model of elimination will be implemented.
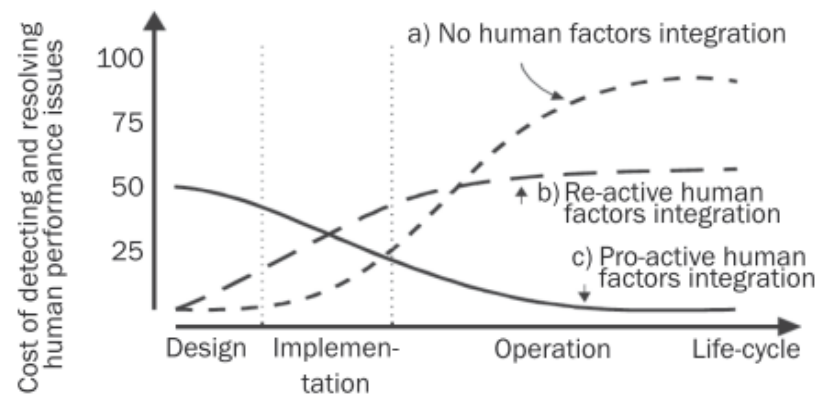

Fig. 5. Cost scenarios of three different life-cycle strategies [21]

With proactive action i.e. study of the cause of errors and with preventive setting of safety systems the trend of error occurrence will decrease. In the field of aircraft maintenance, a number of methods and tools for the identification of the causes of human error and its elimination have been developed.

Human is the key factor in the production process and in the process of operation of technical means since it gives new value to the object of work.

As it can be seen, there is an urgent need for regulations that protect aviation maintenance personnel from pressures and fatigue. These regulations will for sure prevent occurrences in the future that might jeopardize aviation safety [19].

\section{International Survey}

An online international survey has been carried out to figure what is the current status of technical staff. From the beginning there have been several mixed feelings about the participants.

Some of them have refused to answer arguing that they were afraid of consequences.

The participants have been maintenance engineers and technicians, working on Part-145 and Part-M organizations from Portugal, Spain, France, Germany and UK [19]. One of the key points of the survey has been the reduced number of people that agreed to answer; only 49 who have been approached agreed to answer [19]; the ages distribution has been: 7 (14\%) from 18 years old to 25 years old, $13(27 \%)$ from older than 25 years old to 35 years old, 20 (41\%) from older than 35 years old to 55 years old and $9(18 \%)$ older than 55 years old.

Q: How many days you work on a week?

The question about the contracted working days on a week had 49 replies, i.e. 12 (25\%) work 6 days to 7 days in a row and $37(75 \%)$ work 3 days to 5 days in a row on a week [19] as shown in Fig. 6. 


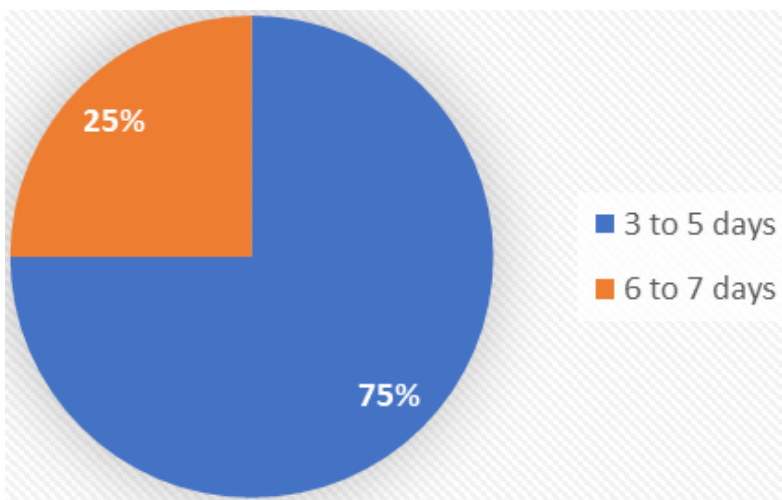

Fig. 6. Contracted working days on a week

Q: How many working hours is your normal working week composed of?

The question about the contracted working hours on a week had 49 replies, i.e., $21(43 \%)$ work more than $40 \mathrm{~h}$ to $50 \mathrm{~h}$ in a week and $28(57 \%)$ work $30 \mathrm{~h}$ to $40 \mathrm{~h}$ in a week [19] as shown in Fig. 7.

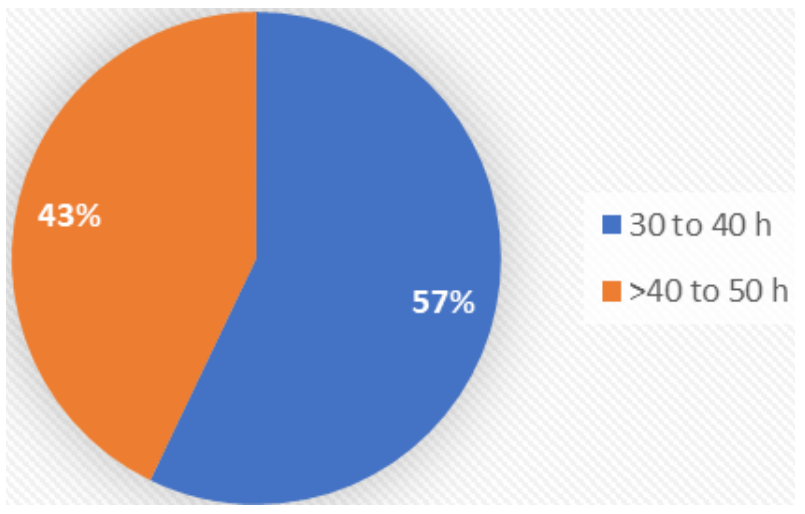

Fig. 7. Contracted working hours on a week

Q: How many extra hours are demanded from your company in a working week?

The question about the extra-hours performed during a working week had 49 replies, i.e., $3(6 \%)$ had no instructions to perform extra hours, $6(12 \%)$ have to perform 1 to 5 extra hours per week, 19 (39\%) have to perform 5 to 10 extra hours per week and $21(43 \%)$ have to perform more than 10 extra hours per week [19] as shown in Fig. 8.

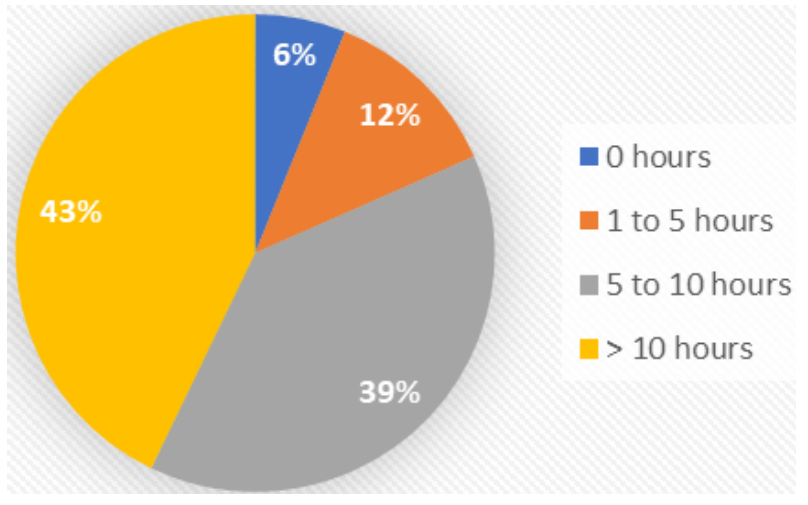

Fig. 8. Demanded extra hours on a week
Q: Are those extra hours paid by your company?

To the question if the extra hours are paid by the company, $28(57 \%)$ answered that they have never received any payment and $21(43 \%)$ have stated that the extra hours performed are paid [19] as shown in Fig. 9.

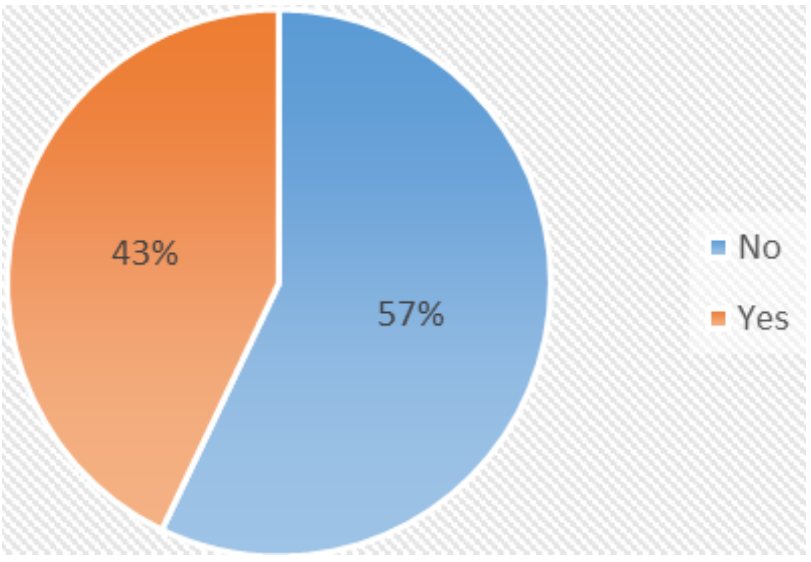

Fig. 9. Paid extra-hours

Q: From your historic background, what were the maximum week working hours ever made?

To the question about the maximum working hours on a week, $5(10 \%)$ answered between $41 \mathrm{~h}$ and $60 \mathrm{~h}, 15$ $(31 \%)$ between $61 \mathrm{~h}$ and $80 \mathrm{~h}$, and $29(59 \%)$ between $81 \mathrm{~h}$ and $100 \mathrm{~h} \mathrm{[19]}$ as shown in Fig. 10.

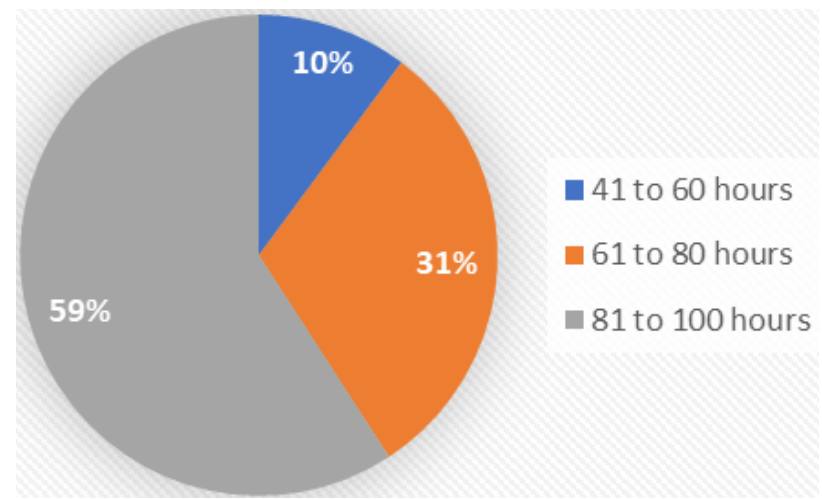

Fig. 10. Maximum historic working hours

Q: How is your physical fatigue after a working week being 0 no fatigued at all and 5 extremely fatigued?

To the question about the physical fatigue felt after a working week, $1(2 \%)$ said that he has very low physical fatigue, $9(18 \%)$ said that they have low physical fatigue, no answers for normal level of physical fatigue, 26 (53\%) have high levels of physical fatigue and 13 (27\%) have very high levels of physical fatigue [19] as shown in Fig. 11.

Q: How is your mental fatigue after a working week, being 0 no fatigued at all and 5 extremely fatigued?

To the question about the mental fatigue felt after a working week, $1(2 \%)$ said that he has no mental fatigue, $1(2 \%)$ has very low mental fatigue, $1(2 \%)$ has low 
levels of mental fatigue, 14 (29\%) have normal mental fatigue, $21(43 \%)$ have high levels of mental fatigue and $11(22 \%)$ have very high levels of mental fatigue [19] as shown in Fig. 12.

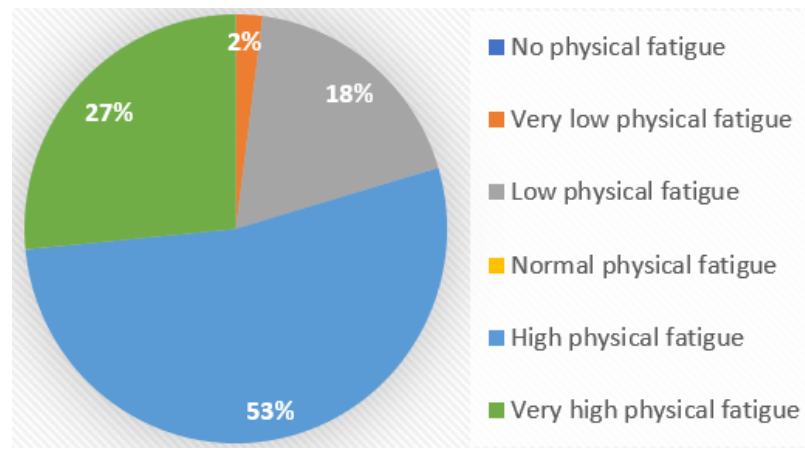

Fig. 11. Physical fatigue

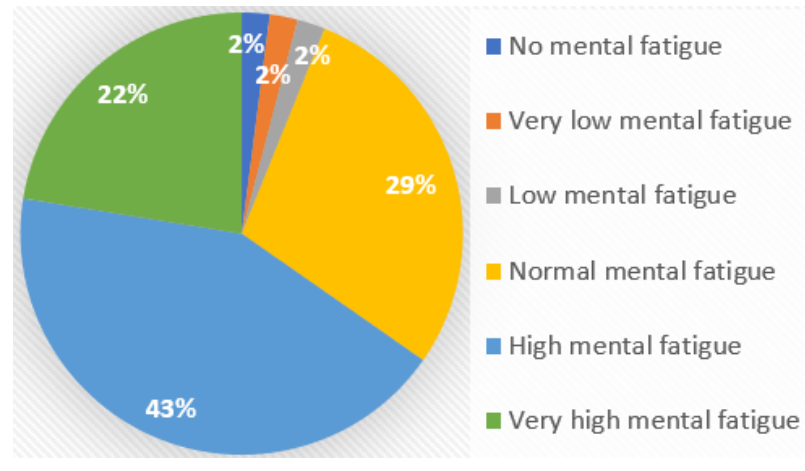

Fig. 12. Mental fatigue

Q: How is your company extra hours' policy?

To the question about the companies' extra work policies, $1(2 \%)$ said that there is no need to perform extra hours, 15 (31\%) said that extra hours are optional, 7 $(14 \%)$ said that it is mandatory to perform extra hours, 8 $(16 \%)$ said that it is mandatory to perform extra hours with a 24 hours' notice and $18(37 \%)$ said that it is mandatory to perform extra hours with a 48 hours' notice [19] as shown in Fig. 13.

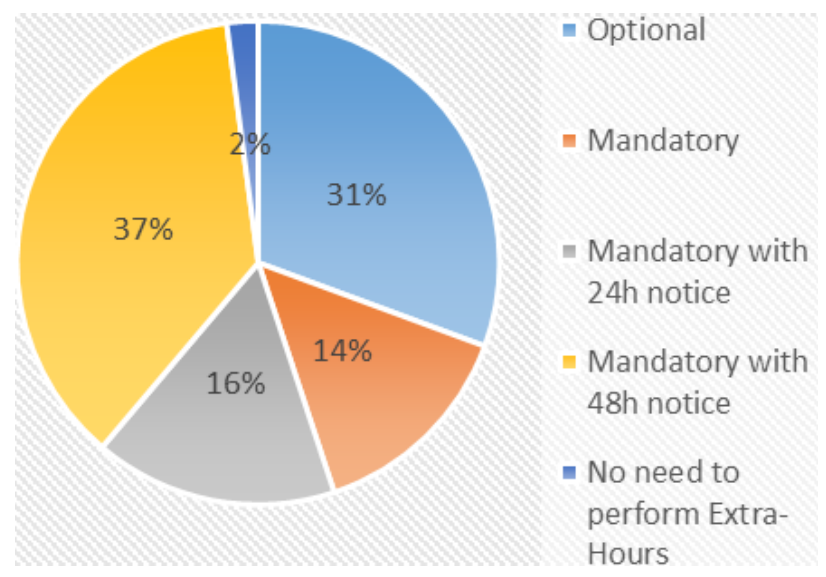

Fig. 13. Companies extra-hours policy
Q: Does your company clearly need the extra hours to perform the contracted maintenance tasks?

To the question if the company needs to perform extra hours to accomplish all the contracted/necessary maintenance works, 4 (8\%) said No and 45 (92\%) said Yes [19] as shown in Fig. 14.

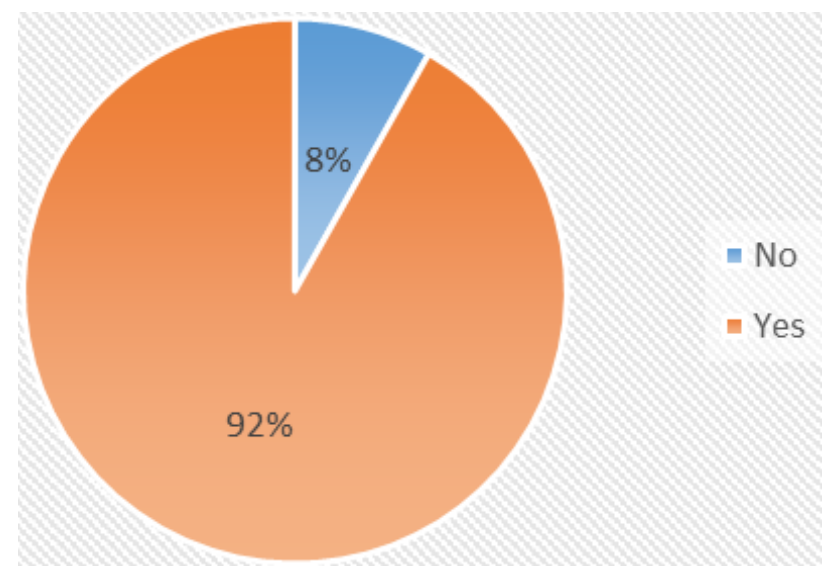

Fig. 14. Companies planning forecast

Q: Do you consider that there is pressure from your company to perform extra hours?

To the question if companies put pressure on their employees to perform extra hours, $6(12 \%)$ answered No and 43 (88\%) answered Yes [19] as shown in Fig. 15.

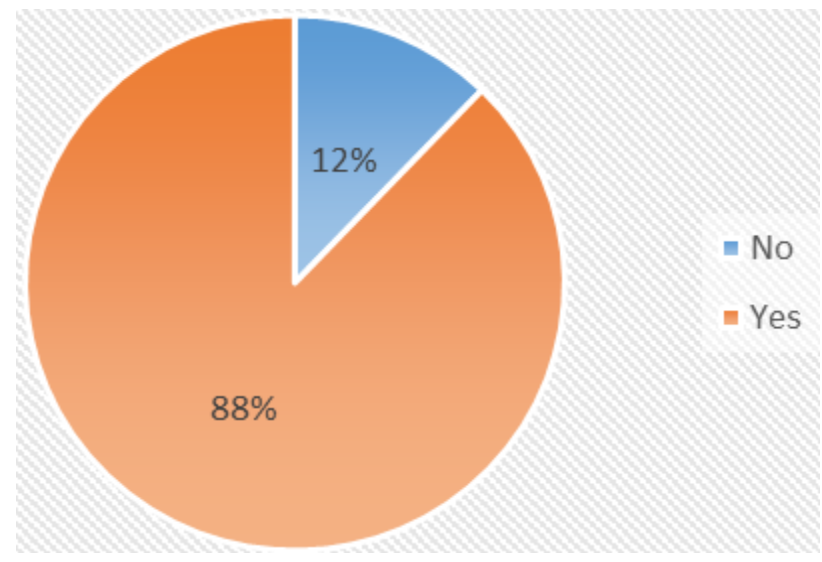

Fig. 15. Pressure to perform extra-hours

Q: Do you consider that your company imposes work schedules that do not allow to rest properly?

To the question if the imposed work schedules does not allow to rest properly, $9(18 \%)$ answered No and 40 (82\%) answered that they do not rest properly [19] as shown in Fig. 16.

Q: Do you have knowledge that someone from your professional network ever made a maintenance error cause by Fatigue or Stress?

To the question if someone has knowledge on their professional network that someone made a maintenance error caused by fatigue or stress, $9(18 \%)$ answered No and $40(82 \%)$ answered Yes [19] as shown in Fig. 17. 


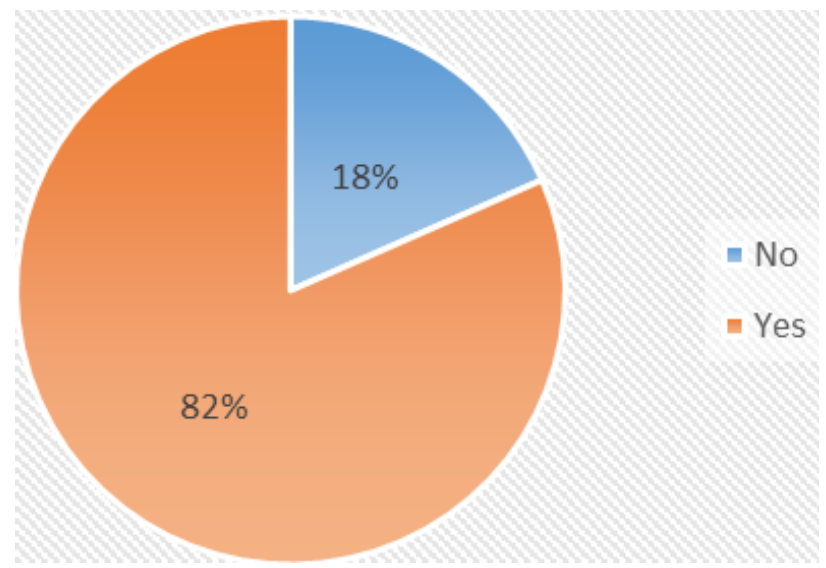

Fig. 16. Companies imposed time schedule do not allow to rest properly

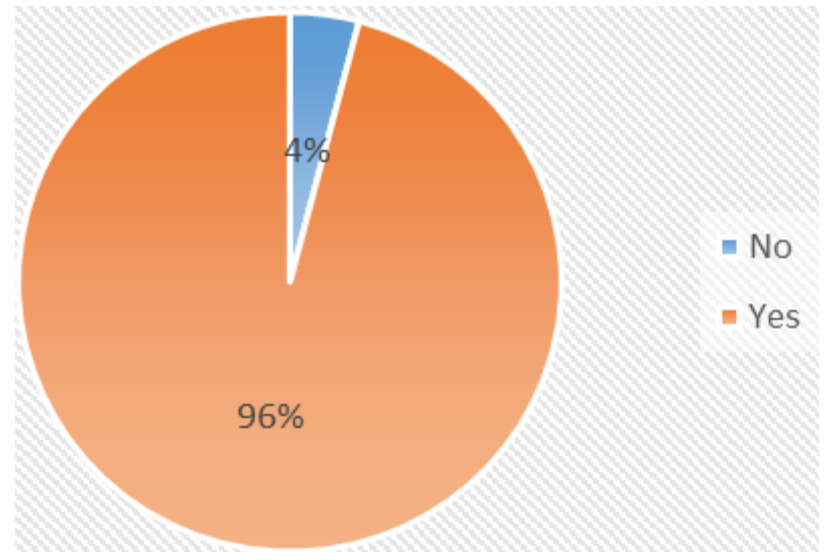

Fig. 17. Maintenance error cause by fatigue or stress

Q: If the answer about the last question is yes, when was the error detected?

To the question, about when eventual maintenance errors made due to stress and fatigue have been detected, $14(36 \%)$ answered immediately, 5 (13\%) answered by an inspector, $14(34 \%)$ answered during tests or final inspections, 7 (17\%) answered with the aircraft in operation [19] as shown in Fig. 18.

Performing a deep analysis of the data, several important factors arise; the most concerning one deals with the age distribution from who made maintenance errors that has been detected only in operation. From these data it can be verified that $4(45 \%)$ were between 25-35 years old, 3 (33\%) were from 35 to 55 years old and $2(22 \%)$ were more than 55 years old [19] as shown in Fig. 19.

The number of extra hours performed by week from who made maintenance errors that has been detected only in operation. From these data it can be verified that $7(78 \%)$ make more than 10 extra hours per week and 2 (22\%) make between 5 and 10 extra hours per week [19], as shown in Fig. 20.

Another significant information is the amount of maintenance personnel that gets paid to perform extra hours and has made maintenance errors that have been detected only in operation. From these data it can be verified that $7(78 \%)$ have not been paid to perform extra hours and $2(22 \%)$ have been paid [19], as shown in Fig. 21.

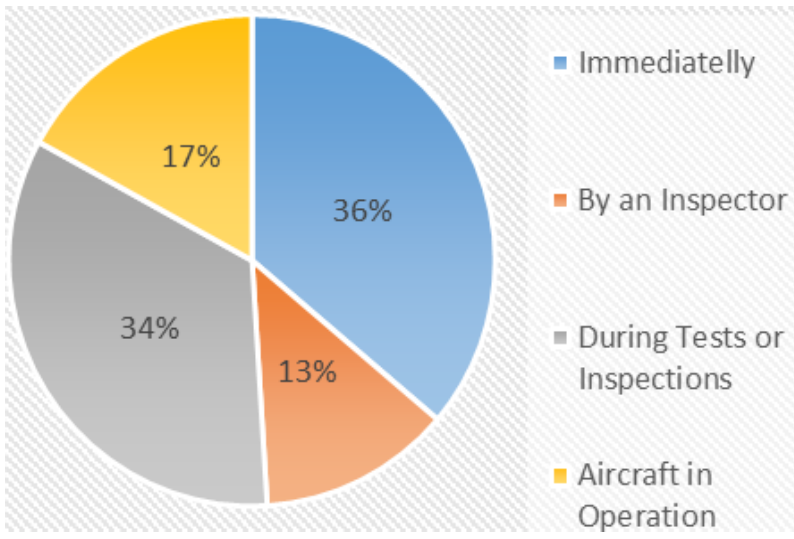

Fig. 18. Maintenance error detection

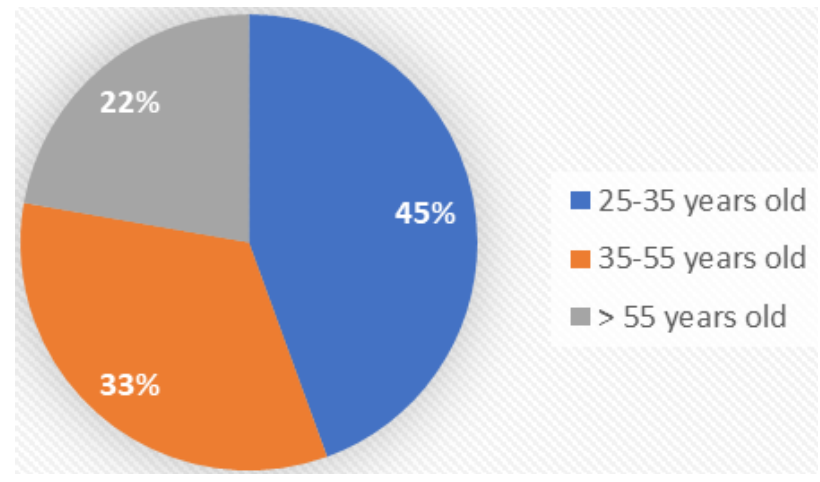

Fig. 19. Age distribution from who made maintenance errors discovered in operation

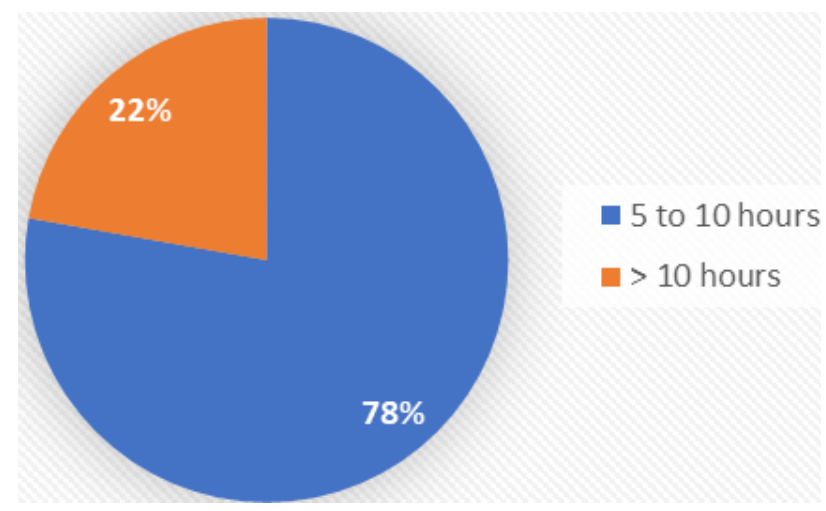

Fig. 20. Extra hours made per week from who made maintenance errors discovered in operation

Some two major concerning aspects arise from the survey [19]. The first one is that companies clearly depend on extra hours to carry out all the contracted maintenance. The Aeronautical regulators are not precise with their instructions regarding the maximum amount of working hours which maintenance staff can perform. The regulations, [12], state that "the companies must ensure the necessary man-power to accomplish the contracted work". As it can be seen, regulators must add additional 
information to [12] in order to clarify the maximum amount of working man-hours.

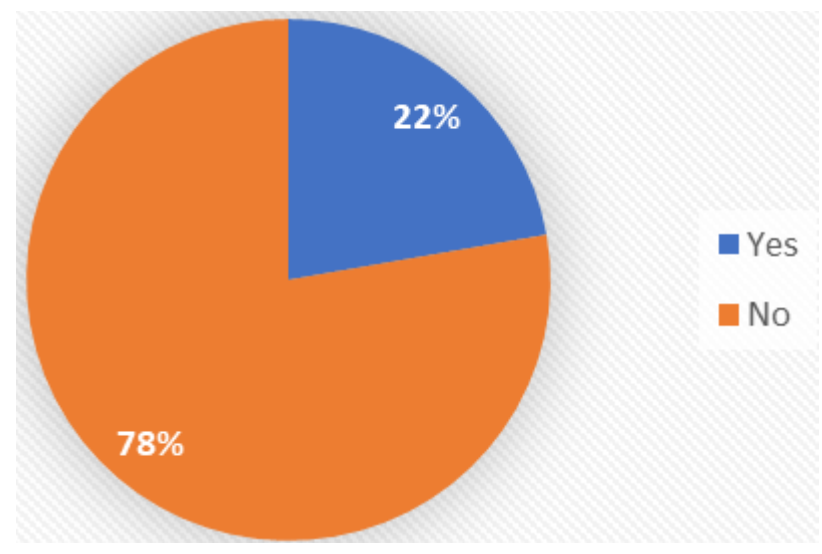

Fig. 21. Number of maintenance personal that get paid and made maintenance errors discovered in operation

The second and most important point, is that $95.9 \%$ has made or knows someone who has made maintenance errors due to stress or fatigue, and from those errors, $17 \%$ has only been detected with the aircraft in operation. This feature is the one who needs to be improved to avoid error propagation up to aircraft operation. Also, $55 \%$ of the maintenance errors discovered in operation has been made by very experienced personnel, which $78 \%$ works more than 10 extra hours per week and only $22 \%$ gets paid for those extra hours. In [22] a similar survey has been performed, and the authors confirmed that also in the Chinese aircraft maintenance companies, regarding the employees, indicated a negative correlation between work stress and job performance. It is already under course an additional survey which will have a more widely adoption and by consequence some more precise results [19].

\section{Hazards Procedures}

Aviation maintenance personnel, subjected to fatigue, stress or pressure, presents hazards procedures which might lead to a potential risk [1], [2], [9], [10], [16], [17], [19], [23]. If pressure related factors, such as time or hierarchic are within the easiest to identify, they are the most difficult to eliminate. Some maintenance organizations, in order to reduce personnel costs, reduce the number of available man-power. Then, in order to compensate the lack of man-power, some companies pay extra-hours, creating the sensation among their employees that it is good for them to work more. Also with these policies, a sentiment is created by the companies that aircraft maintenance jobs are high demanded careers, so maintenance staff needs to be available 24/7 [19]. These working practices are quite common nowadays. Airlines and maintenance markets are under a severe competition; managers tend to stretch teams up to verge and try to mitigate required lack of manpower subcontracting in short term waves. This hazard procedure, engaging in more activities than the available manpower, is not recommended, it is a way to transfer to the maintenance personnel the planning, or the lack of it. According to some interviews, it is also common to assign to the maintenance personal 12 hours or more man-hours a day; the problem is not the delivered assignments, but the extra pressure that is transferred to the maintenance personnel in delivering more workload that is capable of, people tend to speed up the maintenance activities to fit in their time table [19].

Like authors in [24] have stated, in the aviation maintenance environment there are many identifiable stressors. Fatigue caused by working at night and time pressure to get aircraft back into revenue service are two obvious conditions that almost certainly cause stress.

The workload breaks policies, in most of the cases are very strict. Companies tend to apply fix work break periods to optimize production, from the management point of view, these polices are preferable to maintain a constant work flux, nevertheless the maintenance personnel carried is divided into categories, it is not justifiable that a structures technician have the same break rest period as avionics, since working in structures is from the physical point of view much more demanding than working with wirings and electronics [19].

Maintenance and airlines industry are also subjected to events that culminate in AOGs. These events are not predictable. When such events occur, usually there is the need to solve the problems in an expedite timeline.

Usually in such events, there is also an increase in the time period, and in many occasions, employees who are on leave on their resting time must be available in a very prompt time to solve any type of problem. The industry must realize that they should start to plan also for unplanned events, creating dedicated shifts to deal with this constrains [19]. Also, authors in [26] have conclude that among several design factors, the time pressure, the lack of communication, and the fatigue are the main causes for errors in aircraft maintenance. The maintenance workers daily life also affects performance. Sociopathic behaviors reduce significantly human performance, also reduced sleep time to family events or poor personal time planning can jeopardize the human performance [7], [20], [26].

\section{Diagnostics and Recommendations}

The most common fatigue and stress symptoms [23] can be:

Most common symptoms of fatigue:

- Increased reaction time.

- Reduced concentration.

- Diminished memory.

- Mood issues.

- Problem solving routine tasks.

- Micro sleeps.

- Poor decision making.

- Forgetfulness.

- Loss of awareness. 
- Less communication.

- Lack of motivation. Most common symptoms of Stress:

- Agitation.

- Frustration.

- Losing control sensation.

- Mood issues.

- Problems being in a relaxed state.

- Depression.

- Low energy perception.

- Headaches.

- Diarrhea.

- Constipation.

- Insomnia.

- Nausea.

- Grinding teeth.

- Changes in appetite.

- Increase use of alcohol, coffee or drugs.

- Constant worrying.

- Disorganization.

- Poor judgement.

- Inability to focus.

- Avoiding responsibilities.

- Nervous behaviors. Requests from employees can also give indications about their exposure to stress and fatigue [23]. Employers recommendations [23]:

- Build the work schedules to minimize its impact on resting periods.

- Diversify the work breaks from different individual assignments.

- Provide optimum working conditions.

- Implement the use of team resource managements tools to promote awareness to fatigue and stress issues.

- Including on the company SMS a Fatigue Risk Management System.

- Planning the assignments in accordance with the available and contracted manpower.

- Do not deliver more man-hours than the work shift length.

- Making time, financial or other type of pressure to the employees is not recommended.

- Control employees working time, preventing from overworking.

Employees recommendations [23]:

- Create a strict routine of: Good rest quality and sleep patterns; Meals; Type of food.

- Plan the family activities together with the work shifts.

- Discuss the need to be fit for your job with the family.

- On a day off try to maintain the same sleeping and eating routine.

- Monitor the caffeine, nicotine and alcohol since they can interfere with the sleeping routines.

- Avoid heavy meals before bed and night shifts.
- Alert colleagues if any fatigue or stress behavior is detected.

- When feeling tired or sleepy, take a break and report the occurrence.

- When feeling fatigued seek a colleague to dialog and report occurrence.

- Prepare yourself to arrive with enough time to brief about your daily assignments.

- During breaks try to take micro naps, if not possible dialog with colleagues and expose yourself to bright light.

Introducing Fatigue Risk Management System into the companies will increase and expose the awareness to stress and fatigue [10], [17], [23]. These two factors are latent problems on all aeronautical industry. Authorities must embrace this goal, and they should propose or provide guidelines to create both stress and fatigue diagnostics matrix. This matrix should then be implemented by the companies SMS departments to have an early warning about a potential problematic employee [19]. There is an urgent need to create self and external diagnosis and mechanisms. A proposal is the creation of two independent diagnosis matrixes, one for the maintenance personal, other for the FRMS control staff [19]. The purpose of this matrix is to detect fatigue and stress in some situations even before the awareness of the maintenance personal. The implementation of these matrixes could be a huge step forward in the human factors sciences and in the way how maintenance industries focus these problems. The company must enforce the anti-stress and fatigue policies, it must educate managers and supervisors that people are precious assets but they can also be the source of problems if they are not handled correctly. It must be clear that waiting is not a good strategy, stress and fatigue tends to get worse with time, not the opposite.

Teams must be educated to know how to identify the correct symptoms, and when it is the case, ask if they feel fatigued or stressed. In a serene and assertive way, the supervisors should assess together with the worker the source of the symptoms and they should create a plan of action to mitigate or even eliminate the stress/fatigue sources, also, in some situation a medical advice is also recommendable. The key point is to create a healthy environment which decreases the change of maintenance errors that might lead to a potential aircraft problem [1], [2], [9], [10], [16], [17], [19], [23]. There is also a key point that companies must put hard efforts on it, which is their Safety Culture. Employees tend to act like they are told to, so it's quite important to have a strong and widely implemented safety culture. In [27] authors mention that the term 'safety culture' emerged in scientific debates on safety largely after the Chernobyl disaster. The concept is often loosely used to describe the corporate culture in which safety is understood to be the number one priority. In [27] safety culture is analyzed as a 'focused aspect of organizational culture' which defines safety culture as 'the shared and learned meanings, experiences and interpretations of work and safety- 
expressed partially symbolically-which guide peoples' actions towards risks, accidents and prevention'. The "tensions" between safety and economic can be presented as a highly specific and localized part of a more general change in the neo-liberal regulation of aviation, in which privatization, increased competition and commercial interests might put safety cultures to the test. Therefore, it is important to focus on the mutual shaping of safety and organizational culture. Conducting a study similar to the one developed in [7] should also bring new insights about the maintenance personal exposure to stress and fatigue.

\section{Conclusion}

Authorities and regulators must regulate the working time periods for the aircraft maintenance personal like it already happens with Flight Crew and Air Traffic Controllers. It is also advisable that authorities introduce practical measures to fight the Dirty Dozens more proactively. Companies and maintenance personnel must be educated about how to deal with stress and fatigue, and knowing how to identify their symptoms. It is also advisable to companies to create dedicated staff to control such environmental and organizational elements; an SMS department is a very good starting point. Also, it is recommendable to educate all the personnel about the correct actions and behaviors to have on a daily basis to avoid and know how to deal and recognize hazards procedures and activities. It is also suggested to elaborate a diagnosis matrix to help in the identification of symptoms and risky behaviors. An online international survey has been carried out to ascertain the professional levels of the fatigue and the stress and their pressure exposure which had revealed serious concerns, namely, the exposure to stress, fatigue and pressure leads to maintenance errors. Aviation safety relies heavily on maintenance staff. The survey results have revealed serious concerns, namely, the exposure to stress, fatigue and pressure leads to maintenance errors. Serious and firm actions need to be taken by the regulators; companies are decreasing their man-power to save costs and mandating the remaining employees to countless most of them unpaid extra hours causing stress, pressure and fatigue. For the upcoming aeronautical industry 4.0, the digital transformation brought by blockchain technology and internet of things [28] will increase the safety margin with the development of preventive maintenance services, which will then help reducing the aircraft downtime and overhaul safety issues caused by stress and fatigue [29].

\section{Acknowledgements}

This work is funded by: European Union through the European Regional Development Fund, included in the COMPETE 2020 (Operational Program Competitiveness and Internationalization) through the ICT project (UID/GEO/04683/2013) with the reference
POCI010145FEDER007690; Portuguese Funds through the Foundation for Science and Technology-FCT under the project LAETA, reference UID/EMS/50022/2013; Portuguese Foundation for Science and Technology (FCT) under Project UID/EEA/04131/2013.

\section{References}

[1] International Civil Aviation Organization, 2013. Safety Management Manual, Doc 9859 AN/474, third ed., Montréal.

[2] E. Gered, A study of challenges to the success of the safety management system in aircraft maintenance organizations in Turkey, Safety Science, vol. 73, 2015, pp. 106-116.

[3] S. M. O. Tavares, P. M. S. T. de Castro, Maintenance, Damage Tolerance of Metallic Aircraft Structures Materials and Numerical Modelling, Heidelberg: Springer, 2019, pp. 17-22.

[4] D. A. Wiegmann, S.A. Shappell, A human error approach to aviation accident analysis, Ashgate Publishing, UK, 2003.

[5] M. Ward, N. McDonald, R. Morrison, D. Gaynor, T. Nugent, A performance improvement case study in aircraft maintenance and its implications for hazard identification, Ergonomics, vol. 53, n. 2, February 2010, pp. 247-267.

[6] K. A. Latorella, P. V. Prabhu, A review of human error in aviation maintenance and inspection, International Journal of Industrial Ergonomics, vol. 26, 2000, pp. 133-161.

[7] Othman, N., Romli, F., Mental Workload Evaluation of Pilots Using Pupil Dilation, (2016) International Review of Aerospace Engineering (IREASE), 9 (3), pp. 80-84.

doi: https://doi.org/10.15866/irease.v9i3.9541

[8] Aviation Safety Network, https://aviation-safety.net/.

[9] EASA, ICAO Annex 19, Safety management, International Standards and Recommended Practices, European Aviation Safety Agency, July 2013.

[10] A. Hobs, K. B. Avers, J. J. Hils, Fatigue risk management in aviation maintenance: current best practices and potential future countermeasures, DOT/FAA/AM-11/10, Federal Aviation Administration, USA, June 2011.

[11] EASA Part-Air Operations, European Aviation Safety Agency, January 2014.

[12] EASA Part-145, Maintenance Organization Approvals, European Aviation Safety Agency, October 2008.

[13] EASA Part-M, Continuing Airworthiness Requirements, European Aviation Safety Agency, June 2017.

[14] D-AIPX Final Report, Ministère de l'Ecologie, du Développement durable et de l'Energie, March 2016, https://www.bea.aero/uploads/tx_elydbrapports/BEA20150125.en-LR.pdf.

[15] R. C. Graeber, D. A. Marx, Reducing human error in aviation maintenance operations, The Flight Safety Foundation 46th Annual International Air Safety Seminar, pp. 1-7, November 1993, Kuala Lumpur, Malaysia.

[16] G. Dupont, The dirty dozen errors in aviation maintenance, 11th Federal Aviation Admin. Meeting on Human Factors Issues in Aircraft Maintenance and Inspection: Human Error in Aviation Maintenance, pp. 45-49, 1997, Washington, USA.

[17] FAA AMT Handbook Addendum Human Factors, Federal Aviation Administration, 2014.

[18] J. A. Yeow, P. K. Ng, K .S. Tan, T. S. Chin, W. Y. Lim, Effects of stress, repetition, fatigue and work environment on human error in manufacturing industries, Journal of Applied Sciences, vol. 14, n. 3, 2014, pp. 3464-3471.

[19] P. Oliveira, Effects of stress and pressure in high performance employments: aeronautical maintenance. Aeronautical Sciences Thesis Degree, in Portuguese, July 2017, Instituto Superior de Educação e Ciências, Lisboa.

[20] J. C. Taylor, R. L. Thomas III, Toward measuring safety culture in aviation maintenance: the structure of trust and professionalism; The International Journal of Aviation Psychology, vol. 13 n. 4, November 2009, pp. 321-343.

[21] D. Virovac, A. Domitrović, E. Bazijanac, The influence of human factor in aircraft maintenance, Promet-Traffic\&Transportation, vol. 29 , n. 3,2017 , pp. $257-266$. 
[22] Y. Wang, J. C. Keller, Ch. Huang, and R. O. Fanjoy, An exploratory study: correlations between occupational stressors, coping mechanisms, and job performance among chinese aviation maintenance technicians, Journal of Aviation Technology and Engineering, vol. 5, n.2, 2016, pp. 69-80.

[23] FAA Human Factors Operators Manual, Federal Aviation Administration, 2014.

[24] E. Yazgan, M. Ş. Kavsaoğlu, Evaluation of stress affecting aircraft maintenance technician's performance, International Journal of Computing, Communications \& Instrumentation Engineering, vol. 4, n. 1, 2017, pp. 2349-1469.

[25] O. Usanmaz, Training of the maintenance personnel to prevent failures in aircraft systems, Engineering Failure Analysis, vol. 18, n. 7, October 2011, pp. 1683-1688.

[26] Y.-H. Chang, Y.-Ch. Wang, Significant human risk factors in aircraft maintenance technicians, Safety Science, vol. 48, 2010, pp. 54-62.

[27] A. Atak, S. Kingma, Safety culture in an aircraft maintenance organisation: a view from the inside, Safety Science, vol. 49, n. 2, February 2011, pp. 268-278.

[28] N. C. Batista, R. Melicio, V. M. F. Mendes, Services enabler architecture for smart grid and smart living services providers under industry 4.0, Energy and Buildings, vol. 14, 2017, pp. 1627.

[29] European Commission, Industry 4.0 in aeronautics: IOT applications, June 2017, https://ec.europa.eu/growth/toolsdatabases/dem/monitor/content/industry-40-aeronautics-iotapplications.

\section{Authors' information}

${ }^{1}$ Instituto Superior de Educação e Ciências, Lisboa, Portugal.

${ }^{2}$ ICT, Universidade de Évora, Évora, Portugal.

${ }^{3}$ IDMEC, Instituto Superior Técnico, Universidade de Lisboa, Portugal.

${ }^{4}$ Departamento de Física, Escola de Ciências e Tecnologia, Universidade de Évora, Portugal.

Luis F. F. M. Santos was born in Lisbon, Portugal, July 1979. He received the M.Sc. and Ph.D. degrees from the Universidade da Beira Interior, Covilhã, Portugal, in 2009 and 2015, respectively. He is currently an Aircraft Maintenance Engineering Coordinator at OGMA, Invited Assistant Professor at the Instituto Superior de Educação e Ciências, Lisbon and is member of ICT research laboratory at Universidade de Évora, Portugal. His research interests include aircraft maintenance and satellite orientation and stabilization.

Rui Melício IEEE Senior Member. He received the MS degree in electrical and computer engineering in 2004, from Instituto Superior Técnico, Portugal and the $\mathrm{PhD}$ degree in electrical engineering in 2010 from Universidade da Beira Interior, Portugal. He is currently an Assistant Professor with Habilitation at Universidade de Évora and is member of ICT research laboratory at Universidade de Évora; and IDMEC research laboratory at Instituto Superior Técnico, Universidade de Lisboa, Portugal. He as more than 25 years of experience in oil industry. His research interests include: power systems management and operation; power electronics and its applications; renewable energies; smart grids; mechatronics systems and aerospace. He is the author or co-author of more than 250 scientific papers presented at international conferences or published in reviewed journals. 